\title{
Verzeichnis der Redner.
}

Urbertt, Dr., 2 Wtesbaben $23-27,34, \begin{array}{r}\text { Seite } \\ 64,73,74,76-78,79,80,81,82, \\ 124,138,144 / 45,164,165,182,183, \\ 191,279,306 / 07\end{array}$

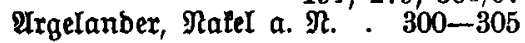

Balfe, Salle a. S. . . . . . 91

Behrend, ßojen . . . . . 30

Berger, sörltb. . . . . 229-240

Bertbols, Blumentyal t. Shan. 82/83, $88,96,99,109,111 / 12,114,116$, $123 / 24$

Bomf包tex, Mtatnz . . . . . 74

Brobfúbrex, Berltn . . . 257-263

Burmetiter Berlin . . . 32, 114/15

Eogn, \$avelberg . . . 253/54, 257

Creceltus, Charlottenburg 124-138, $143 / 44,227,317-325$

(röв́mann, Rulmbađ) . . . 330

Erliger, Dr., EharIottenburg 15, 34-64, $81 / 82,95 / 96,112,116-123,138-140$, $146-164,182,183-190,192-206$, 217 , 226, 227, 254/55, 297/98, 305, $326,327,328$

Dörx, Frtebberg a. 5 . . . . . 326

Glteles, ßofen. . . . . . . 30

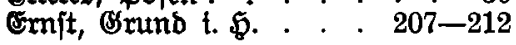

Feterabend, Diundjen 89-91, 212-214

₹elbheim, Purg b. M. $79-81,243$,

$253,254,256,257,263,276,279$,

280, 297, 299, 305, 317, 326, 327, 331

Bennes, Darmjtabt . . . . 30/31

Gruner, \$for 5 betm . . . . 215/16

(Stintber, Sajtatt. . . . . . 330

(Sutjamtbt, ફavelberg . . . . 253

Đäfner, IXten a. \&. . . . 255/56

Janjen, Rter. . . 94/95, 109-111

ఫeuzerotb, Meiningen . . . 330
Seite

Wunerasły, \$ojen. . . . . . 32

Jaredt, ßofen . . . 264-276, 279

Rtrid, Rarlsిrube i. B. . . . . 241

Ritnzer . . . . . . . . 28/29

Ruxz, Stettin . . 243-253, 256, 307

Detfifert, Rarlsrube 1. $\mathfrak{B}$. . . 328/29

Mader, Mündien . . . . . . 92

Mautber, Rarlsathe t. $\mathfrak{B}$. . . 240/41

Ftolte, \&ubenifieto 191/192, 212, 215 , $217,224,225,228,240,241,242,243$

D西, Sotha . . . . 164/65, 214/15

\$inct, Meł . . . . . . . . 91

Mabethge, Berlin . . 84-88, 97-99

Mente, Đannover . . . . 280-296

Fequabt, \&utben . . . . . . 299

Fumpf, SBiesbaden . . . . 140/41

Sđjetot, Perltn. . 28,92-94, 115/16

Sqututng, \$ojen . . . 96,112-114

Snnetber, Dr., Botsbam 226/27, 228

Shoebon, SIetritb . . . . 328

Sauter, Bmetbrituen . . . 65-73

ङ中ulb, హttlebne . . . . 165-182

Sqmarstopff, D. Dr., ßojen 27/28

Stmon, Sofen ...... 14, 21

Soerenjen, Bexlin . . . . 327/28

Sttben, BerItn . . . . . 74-76

§wart, Dr., ßo「en . . . . . 33

Tems, BexItn . . . . . . $\mathbf{3 3 / 3 4}$

Treuner, Dr., Berlin . . 277-279

Malbecter, Dr., Eyarlottenburg 99-109

F̧elă, Brešlaut . . 224/25, 241/42, 331

Wenmuth, $\mathfrak{E a f f e l}$. . . . . 97, 331

Wriglow, Branbenburg a. $\tilde{y} . . \quad 329$

Witms, \&ubenictetb 218, 224, 329/30

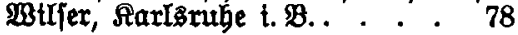

Btebe, Pofen . . . . . 141-143 
